\title{
The Teaching Strategies of Chinese Compound Words for Korean Students
}

\author{
Yaxi Jin \\ College of Advanced Chinese Training, Beijing Language and Culture University, Beijing, China \\ gimahi1980@163.com
}

Keywords: Chinese teaching for Korean students; Compound words; Parataxis; Semantic model

\begin{abstract}
According to the mother tongue transfer theory, vocabulary comparison can effectively find out the difficulties in the teaching of vocabulary in teaching of Chinese as a foreign language, and seek the corresponding teaching strategies. The most obvious contrast between Chinese compound words and Korean compound words is parataxis and hypotaxis. The main semantic model of Chinese compound words is 'Suggestive feature + category'. There are three types Chinese compound words which are difficult to acquire for Korean students: 'nominal morpheme + verbal morpheme' type compound nouns, compound words of nominal morpheme as adverbial, compound words including semantic redundant element. To carry out the concept of the main semantic model in teaching can help students to process lexical informations more accurately and effectively.
\end{abstract}

\section{对韩国学生的汉语复合词教学策略

\author{
金娅曦 \\ 北京语言大学 汉语进修学院，中国 北京 100083 \\ gimahi1980@163.com
}

摘要: 根据母语迁移理论, 词汇对比可以有效地发现对外汉语词汇教学中的难点, 并寻求相 应的教学对策。“意合”与 “形合” 是汉 - 韩复合词最显著的对立特征, 汉语复合词的主要语 义模式是 “提示特征+事物类别”。“动名” 型复合名词, 名语素作状语的状中式复合词, 以及 语义上含冗余成分的复合词是韩国学生习得的难点, 在教学中贯彻主要语义模式的概念, 能 帮助学生更为准确有效地进行词汇信息加工。

关键词：对韩汉语教学；复合词；意合；语义模式

\section{1. 引言}

在对外汉语教学中, 词汇教学不但是基础性环节, 还具有持久性, 向来是对外汉语教学的重 点。随着母语迁移理论受到越来越广泛的关注和重视, 词汇教学有了向国别化发展并结合语 言对比的需求, 对此, 周小兵, 钱芳, 裴德成 (2012) 也指出 “根据对比可以设计出有效的 教学方法, 帮助学生掌握好汉语词汇的意义和用法”。近年来, 对韩学生的词汇教学研究也取 得了一定的成果, 主要集中在偏误词汇教学和汉源词的对比研究部分。本文以汉语词汇中占 绝大部分的复合词的教学为研究对象, 通过汉・韩两种语言复合构词法的对比, 凸显出汉语 复合词的特点及教学中的难点, 结合汉语复合词的主要语义模式, 提出相应的教学策略。

\section{2. 汉・韩复合词对比}

2.1. 构词语素

2.1 .1 复合构词法与派生构词法的兼容性

汉语复合词由词根和词根组成, 词根可以是复合词, 但不能包含词缀, 也就是说汉语复合词 
的语素没有派生形态, 汉语的复合构词法和派生构词法相互排斥, 不可能共同完成构词。汉 语动名型复合名词的构词成分就是动词性词根和名词性词根, 如 “耕地, 来信, 跳马, 跑鞋, 跑道，煎饼，冠军，触摸屏”等。

韩语复合词由词基和词基组成, 词基虽然是单纯词, 但可以包含派生词缀, 即是说词基可以 是（临时性）派生词, 韩语复合词可以由复合构词法与派生构词法共同作用形成, 构词语素 先派生再复合。就韩语动名型复合名词而言, 动语素添加名词化词缀后再与名语素复合的情 况非常普遍，如:

nuleumdanchu（nuleu+m+danchu, 按 + 名词化词缀 + 扣子=按钮）

noliteo（nol+i+teo，玩+名词化词缀+地方=游戏的地方）

2.1.2 词内切分

汉语复合词由词根和词根组成，韩语复合词由词基1和词基或比词基更大的语言单位（即能独 立运用的词）组成，二者的直接组成成分都是两个，但是词内切分却不尽相同。

汉字都是一字一音节, 并且绝大部分汉字都有独立完整的语义, 一般来说汉语复合词的一个 音节就是一个词根，所以汉语复合词以双音节词居多。而多（两个以上，下同）音节词一般 也是多词根的情况, 但是直接组成成分仍然是两个, 即是说, 可能其中一个组成成分或两个 组成成分本身就是复合词，如: “丹顶鹤，复印机，高等教育”等。

与之相比, 韩语的一个音节不像汉字那样都具有独立的意义, 因而一个词基通常并非只有一 个音节，所以韩语复合词中双音节词的比例并不占优，甚至处于劣势，但是构词词基只有两 个，不像汉语复合词那样存在多词根的情况。

表 1 汉 · 韩复合词内部结构对比

\begin{tabular}{|l|l|l|l|l|}
\hline 复合词 & 语素 & 可否包含派生形态 & 音节 & 直接组成成分 \\
\hline 汉语 & 两/三个词根 & 否 & 双音节为主 & 二分 \\
\hline 韩语 & 两个词基 & 是 & 多音节为主 & 二分 \\
\hline
\end{tabular}

\section{2. “意合”与 “形合”}

汉语复合构词法与句法有相当的一致性，对此，刘丹青（2008:584）曾指出：“复合词实际上 使用类似句法 (而不是形态) 手段来构造新词”，这也是汉语学界一贯的认识。韩国语的复合 词根据语素间的语序与连接形态 2 是否符合句法规则，分为句法复合词与非句法复合词 3 ，虽 然因为不同学者区分标准上的差异，统计结果不尽相同，但 “句法复合词” 都大大多于 “非 句法复合词”，说明韩语的复合构词法也与句法基本一致。既然汉・韩语的复合构词法都与句 法基本一致, 从理论上来说, 两种语言的复合构词法的差异也应该取决于两种语言句法的差 异。奈达 (E.Nida)（1982:57）曾经指出：“就汉语和英语而言，也许在语言学中最重要的一 个区别就是 “意合”与 “形合” 的对比。”按类型学的划分, 汉语属于形态极不发达的 “孤立 语”，而韩语属于形态高度发达，比英语还发达的 “黏着语”，所以 “意合”与 “形合”也正 是汉语和韩语的句法特点。通过对两种语言复合构词法体系的考察, 我们发现这也正是两种

\footnotetext{
${ }^{1}$ 是词根和词干的总称, 指在某一词法操作中加上某个词缀之前的形式, 可以包含全部或部分派生词缀。汉语的形态单一, 词根、词干和词基重合，实素等同于词根，在讨论复合构词法时只用 “词根” 这一概念。

2连接形态主要指名词化词尾 “(으), 이, 기, 개” , 连接词尾 “아/어” 和定语化词尾 “는/(으)ㄴ/” 的使用情况。

对于句法复合词和非句法复合词的判定标准，各家的意见不尽相同。

허응 (1975) 对句法复合词的划分标准最为宽松, 他认为: “除了两个词干间不添加任何词尾, 就直接相连的情况外, 都 属于句法复合词。” 김정은 (1995) 对句法复合词的划分标准也比较宽松, 他认为只要语素的语序和连接形态合乎短语的 构成方式, 无论发生音变与否都属于句法复合词。李翊㷏等 (2008) 的划分标准就严格得多, 他们认为即使语素的排列顺序 和连接形态符合句法要求, 只要组成语素发生音变, 包括韵脱落、添加、改变, 如果发生了音变就应该属于非句法复合词, 但因为添加属格助词而发生音变的情况除外。金镒炳 (2000) 的划分最细致, 标准也最严格, 他认为只要发生了音变, 属格 型音变也包括在内, 均属于非句法复合词。

本文认为语音变化本不在句法讨论的范围内, 所以也不应该影响句法复合词的判定，所以采用仼不合（1995）的划分标准
} 
语言复合构词法的最大区别特征, 这一点从二者的分类体系标准上就一览无余。 对汉语复合构词法最通行的分类就是从语素间的结构关系入手, 分为 “并列式 4 , 定中式, 补 充式, 动宾式, 主谓式” 五大类, 而甚少关注形态。首先, 这种分类不能确定, 也并不关注 复合后的词性 5 ; 其次, 不考察组成语素各自的词性和搭配组合; 再者, 构成语素间没有任何 形态上的连接手段。而韩语复合构词法则不同, 极其关注形态, 体现在各个层级的分类标准 中。第一层级 “句法复合词” 和 “非句法复合词” 的分类标准就在于语素间的语序与连接形 态是否符合句法规则。第二层级的分类依据复合词的词性, 分为 “复合名词, 复合动词, 复 合形容词, 复合副词” 等几个次类, 而且整个复合词的词性就取决于后一语素的词性（除了 极少数后一构词语素是不完整语基的复合词)。第三层级的分类是按照构词语素词性的组合方 式, 如 “名词 + 名词”、“动词词基 + 连接词尾 + 动词” 等。与汉语不同, 语素间的语义关 系并非韩语复合构词法主要关注的内容, 也较少据此进行系统分类, 一般只是在讨论 “名词 + 动词” 式复合动词时会区分名词是主语还是宾语, 这是因为韩国语属于 SOV 型语言, 在缺乏 格助词的情况下不能确定名词和动词的语义关系。

\section{3. 汉语复合词的主要语义模式}

董秀芳（2005：129-142）指出汉语复合名词的主要语义模式 “提示特征 + 事物类”，复合 动词的主要语义模式 “方式或途径 + 行为或结果”, 并进一步指出, 二者在一个抽象的层次 上具有一致性, 因为行为的方式也是对行为进行分类的提示特征, 而行为或结果也属于广义 的事物。我们同意她的观点, 并且鉴于复合名词和复合动词占据了汉语复合词的绝大部分, 我们认为 “提示特征 + 事物类” 也代表了汉语复合词的主要语义模式。“事物类” 和 “提示 特征” 都是高度概括的概念, 前者是我们认知客观世界时划分出的各种范畴, 既包括实物, 又包括抽象的事物, 又包括行为和结果; 后者更是保罗万象, 只要能帮助我们确定认知对象 的都可以成为提示特征, 颜色、形状、气味、数量、用途、加工过程、方式、途径、材料、 施事、受事、行为、活动范围等都包括其中。

语义模式不仅是对汉语复合词语素语义关系的归纳, 还能跨越句法的制约, 主导词汇信息加 工, 甚至形成自主词法结构。对于汉语这样重 “意合” 的语言来说, 在缺乏句法和形态标记 辅助的情况下, 灌输语义模式的概念对攻克词汇教学中的难点尤为有效。

\section{4. 复合词教学中的难点及其教学策略}

4.1. “动名” 型复合名词

在汉语的复合词中, 有一部分由 “及物动词语素 + 名语素” 构成动名型复合名词, 只有名词这 一种词性, 如: “食物, 动物, 发票, 炒饭, 转盘, 试卷, 摇篮” 等。这些 “动名” 结构中的 前一语素只有动词的义项, 但在信息加工时我们没有处理为行为动作, 而是处理为后一事物 的提示特征, 动词的功能从述谓降级为指别。汉语复合词的主要语义模式使得这类动名组合 跳脱句法规则的制约, 不能被解读为述宾式动词短语。此外, 还有一部分动名型复合名词是 动名兼类, 如 “耕地, 烤鸭, 煎饼, 赔款, 包车, 刹车, 得分” 等, 但在没有语境提示的情 况下, 我们仍然优先把它们处理为名词, 这也可谓是这一语义模式主导词汇信息加工的又一 例证。

4.2. 名语素作状语的状中式复合词

\footnotetext{
${ }^{4}$ 由两个意义相同、相近、相反或相关的词根并列组合而成。合成后的词义可能和原来的两个词根相同, 如: 途径, 收获, 价值等; 也可能发生变化, 如: 骨肉, 领袖, 大小等; 抑或后一个词根的意义完全消失, 如: 国家, 质量, 动静等。所以虽 然被称为并列式, 但前后两个语素的意义不一定平等。北大版《现代汉语》 (2004：197) 认为并列式复合词在意义上前后两 个语素地位平等, 本人不同意此观点, 当后一词根意义完全消失时, 地位完全弱于前一词根。

5通行的语法著作中, 一般只是把 “定中式” 和 “补充式” 进一步划分为名词性和动词性两个次类, 只有朱德熙 (1982) 进 一步列出了各种结构关系下存在的多种复合词词性类别。
} 
在现代汉语的句法结构里, 名词的原型功能是作主语和宾语, 作状语则被视作 “非范畴化” 的表现, 所以名语素作状语的复合词也被划归广义的非句法复合词, 包括复合动词和复合形 容词两类, 如 “瓜分, 鲸吞, 䖯食, 鞭策, 网罗, 化疗, 雪白, 冰凉” 等。虽然名词作状语 在古代汉语里相当常见, 但是在现代汉语句法中却基本消失, 所以如果简单套用现代汉语的 句法来分析的话, 这类词应该被解读为主谓式复合词, 但是我们却能正确地把它们理解为状 中复合词, 这也得益于主要语义模式的主导作用。可见, 汉语复合词的主要语义模式不但对 汉语复合词语素间的语义关系有很强的解释力, 而且能主导汉语使用者对词汇信息的加工, 特别表现在上述一些从句法的角度难以解释的词上。在对非句法复合词词义的信息加工上, 主要语义模式的影响要强于句法规则的影响。

4. 3. 含冗余成分的复合词

Noyer (1997) 提出 “词法具有一个自主的结构, 一个词必须满足这个自主结构的某些要求才是 合法的。而词法自主结构是因语言而异的, 每个语言的词法可能规定了不同的 “词层面完好 构造条件”。” 就汉语而言 “提示特征 + 事物类” 不但是汉语复合词的主要语义模式, 也是潜 在的自主词法结构, 某些构词成分的出现仅就是为了满足这种词层面的完好构造条件, 从语 义上来说是冗余成分。如:

机打一一用机器打印, “机” 是工具, 但打印一定是用机器, 机打=打 (印), 所以 “机” 沦为 语义上的咒余成分。

记住一一通过记忆使之停留, “住” 是结果, 但 “记” 的语义本身也已经包含了 “住” 的结果, 记住=记, 所以 “住” 沦为语义上的先余成分。

此外, 汉语的外来词也非常有特点。每一种语言的词汇都存在外来语, 大部分语言对外来语 采用音译的方式借入, 而汉语则一般要从语音和构词法上进行改造, 用复合词法对源语言进 行加工, 比较典型的做法的就是 “音译 + 意译”。如 “啤酒 (beer + 酒), 酒吧 (酒 + bar), 卡车 $(\mathrm{car}+$ 车), 卡片 $(\mathrm{card}+$ 片), 艾滋病 $($ AIDS + 病), 香槟酒 $($ 香槟 + 酒 $)$, 三文鱼

$($ salmon + 鱼), 夹克衫 $($ jacket + 衫 $)$, 芭蕾舞 $($ ballet + 舞 $)$, 法国 (France + 国)”等。 这些外来词的构词实际上也是遵循了 “提示特征 + 事物类” 的语义模式, 音译部分就是提示 特征, 提取自源语言的全部或部分发音, 最后添加的意译部分就指出了事物的类别, 从意义 的识别上来说是咒余成分。这类复合词的构词成分中都有一部分是语义上的午余成分, 势必 形成学生理解上的障碍, 但如果引入汉语复合词主要语义模式的概念, 让他们明白语义上的 咒余成分是汉语自主词法结构的构词需要, 这些难点将会不攻自破。

\section{5. 结论}

在对外汉语的词汇教学中结合词汇对比能准确发现教学难点, 并制定有效的教学策略。通过 对汉 - 韩复合构词法的对比, 我们发现二者在词语内部结构上存在异同, “意合” 与 “形合” 是二者在构词上最为突出的对立特征。汉语复合词的主要语义模式是 “提示特征+事物类别”, 它不仅是对汉语复合词主要语义关系的概括, 还能主导词汇信息加工, 甚至形成了潜在的自 主词法结构。基于汉语复合词注重语义的特点, 我们应该充分利用主要语义模式来推进教学。 对韩国学生来说, “动名” 型复合名词, 名语素作状语的状中式复合词, 以及语义上含冗余成 分的复合词是教学中的难点, 汉语复合词主要语义模式的概念会对学生的词汇信息加工形成 良好的引导, 有助于教学难点的攻克。

\section{6. 致谢}

本课题得到北京语言大学 2016 年度院级科研项目 “汉韩复合构词法对比” (项目批准号 16YJ080204）经费支持, 特此致谢。 


\section{参考文献}

[1] 周小兵, 钱芳, 裴德成. 韩汉对比与对韩汉语教学——兼论《怎样教韩国人学汉语》. 汉 韩语言对比研究(3).第四届汉韩语言对比国际学术研讨会论文集[C]. 北京: 北京语言大学 出版社,2012: 12-26.

[2] 北京语言大学中文系现代汉语教研室. 现代汉语[M]. 重排本. 北京: 商务印书馆, 2004.

[3] 刘丹青. 语法调查研究手册[M]. 上海: 上海教育出版社, 2008.

[4] 허응. 우리 옛말본[M]. 샘문화사, 1975.

[5] 김정은. 국어단어형성법연구[M]. 박이정, 1995.

[6] 李翊鲚, 李相亿, 蔡琬(著). 张光军, 江波(译). 韩国语概论[M]. 北京：世界图书出版社, 2008.

[7] 金镒炳. 국어합성어연구[M]. 역락, 2000.

[8] NIDA EUGENE A. Translating Meaning [M]. San Dimas, California: English Language Institute, 1982.

[9] 朱德熙. 语法讲义[M]. 北京：商务印书馆, 1982.

[10]董秀芳. 汉语的词库与词法[M]. 北京: 北京大学出版社, 2004.

[11]NOYER ROBERT ROLF. Features, Positions, and Affixes in Autonomous Morphological Structure [M]. New York \& London: Garland Publishing Inc. 1997.

\section{Acknowledgement}

This research was financially supported by the 2016 Research Project of Courtyard's Level Item in Beijing Language and Culture University (16YJ080204)

\section{References}

[1] Zhou Xiaobing, Qian Fang, Pei Decheng. Comparison of Korean and Chinese and the Teaching of Chinese to Korean — also on <How to Teach Korean to Learn Chinese>. The Contrastive Study of English and Chinese (3). Proceedings of the Fourth International Symposium on Chinese and Korean language[C]. Beijing: Beijing Language and Culture University Press, 2012.

[2] Department of modern Chinese language and literature, Peking University. Modern Chinese [M]. Revised Edition. Beijing: The Commercial Press, 2004.

[3] Liu Danqing. Handbook of Grammar Investigation [M]. Shanghai: Shanghai Education Press, 2008.

[4] HeoEung. Ancient Korean Grammar [M]. Saemmunhwasa, 1975.

[5] GimJeongeun. Korean Word Formation Studies [M]. Bagijeong, 1995.

[6] Li Yixie, Li Xiangyi, Cai Wan (write). Zhang Guangjun, Jiang Bo (translate). Korean Studies [M]. Beijing: World Books Publishing House, 2008.

[7] Gimlkbyeong. Korean Compound Words Studies [M]. Yeoglag, 2000.

[8] NIDA EUGENE A. Translating Meaning [M]. San Dimas, California: English Language Institute, 1982.

[9] Zhu Dexi. [M]. Beijing: The Commercial Press, 1982.

[10]Dong Xiufang. Chinese Lexicon and Morphology [M]. Beijing: Peking University Press, 2004. 
[11]NOYER ROBERT ROLF. Features, Positions, and Affixes in Autonomous Morphological Structure [M]. New York \& London: Garland Publishing Inc. 1997.

作者简介: 金娅曦 (1980-), 女, 云南, 讲师, 主要研究语言对比与对外汉语教学, gimahi1980@163.com。 